\title{
Functional Connectivity Separates Switching Operations in the Posterior Lateral Frontal Cortex
}

\author{
Christine Stelzel $^{1,2}$, Ulrike Basten ${ }^{1,3}$, and Christian J. Fiebach ${ }^{1,3}$
}

\begin{abstract}
Task representations consist of different aspects such as the representations of the relevant stimuli, the abstract rules to be applied, and the actions to be performed. To be flexible in our daily lives, we frequently need to switch between some or all aspects of a task. In the present study, we examined whether switching between abstract task rules and switching between response hands is associated with overlapping regions in the posterior lateral frontal cortex and whether switching between these two aspects of a task representation is neurally implemented by distinct functional brain networks. Subjects performed a cue-based task-switching paradigm where the location of the task cue additionally specified the response hand to be used. Overlapping activity for switching between abstract rules
\end{abstract}

\section{INTRODUCTION}

Cognitive flexibility is essential for meeting the multitasking requirements of our environment. This includes permanent switching between stimulus information, between tasks, and between actions, being particularly demanding when there is high similarity between tasks, for example, because of overlapping stimulus or response categories (Brass et al., 2003; Schuch \& Koch, 2003; Meiran, 2000). Switching between two tasks has been associated with activity in the lateral frontal cortex (Braver, Reynolds, \& Donaldson, 2003; Brass \& von Cramon, 2002; Dove, Pollmann, Schubert, Wiggins, \& von Cramon, 2000). In particular, a region in the posterior lateral frontal cortex at the intersection of the inferior frontal and precentral sulci, now frequently labeled as inferior frontal junction (IFJ) area, has been associated with the updating of task representations in task switching, a process that allows us to adjust our behavior in advance to a new task environment (Brass, Derrfuss, Forstmann, \& von Cramon, 2005). In addition, the IFJ has been shown to be involved in further task contexts involving the updating of representations at different representational levels, such as in the Stroop task or the $n$-back paradigm (Derrfuss, Brass, Neumann, \& von Cramon, 2005; Derrfuss, Brass, \& von Cramon, 2004).

\footnotetext{
${ }^{1}$ University of Heidelberg, ${ }^{2}$ Charité Universitätsmedizin, Berlin,
} Germany, ${ }^{3}$ Goethe University Frankfurt versus response hands was present in the inferior frontal junction area of the posterolateral frontal cortex. This region, however, showed very distinct patterns of functional connectivity depending on the content of the switch: Increased functional connectivity with anterior prefrontal, superior frontal, and hippocampal regions was present for abstract rule switching, whereas response hand switching led to increased coupling with motor regions surrounding the central sulcus. These results reveal that a rather general involvement of the posterior lateral frontal cortex in different switching contexts can be further characterized by highly specific functional interactions with other task-relevant regions, depending on the content of the switch.

This suggests that the role of the IFJ in updating task representations might be more general, including different levels of a task representation, such as stimulus and response representations (e.g., in the Stroop task) as well as abstract task rule representations in situations where stimuli and responses remain the same (e.g., in the taskswitching paradigm). However, a direct comparison of activity and connectivity patterns of the IFJ for switching between different levels of task representations within one paradigm, which would provide the most direct evidence for an overlap of functionality in this region, was not yet undertaken.

In the present study, we used a cue-based task-switching paradigm to directly compare activity changes associated with the switching between abstract task rules, that is, rules that specify how identical stimuli are mapped to identical responses on the basis of a specific categorization and activity changes during switching between response rules, that is, rules that only differ with respect to the response set while stimuli and abstract rules are identical. Within one fMRI session, participants performed two runs of a task-switching paradigm. The identity of the task cue (i.e., square or diamond) informed participants which abstract task rule is relevant on the next trial (Rogers \& Monsell, 1995), the location of the task cue (i.e., left or right of a fixation cross) indicated which response hand to use in the next trial. That way, we were able to investigate within one paradigm whether switching between abstract rules 
and switching between motor responses activates overlapping regions in the IFJ. The finding of functional overlap in the IFJ would provide most direct evidence for a general function of the IFJ in the activation of different aspects of a task set, ranging from specific motor parameters up to abstract rule representations.

To elucidate the mechanisms on how the IFJ updates task representations, we also assessed the functional connectivity of the IFJ during abstract rule and response switching, respectively. In particular, we tested whether switching is associated with differential connectivity patterns of the IFJ region, depending on the content of the switch. Up to now, little is known about the specific pattern of connectivity of this region related to the updating of task representations. As previous studies found taskswitching-related lateral frontal activity not only in the IFJ but also in more anterior prefrontal regions (Forstmann, Brass, Koch, \& von Cramon, 2006; Braver et al., 2003), we predicted IFJ activity during abstract rule switching to be characterized by interactions with regions in anterior portions of the lateral PFC. Likewise, on the basis of previous reports of strong anatomical (Pandya \& Barnes, 1987; Barbas \& Mesulam, 1985) and functional (Rowe, Stephan, Friston, Frackowiak, \& Passingham, 2005; Stephan et al., 2003) connectivity of frontal regions with more posterior motor-related regions during spatial and motor tasks, we expected functional connectivity of the IFJ with these posterior regions to be stronger during switching between motor responses. The finding of a differential connectivity pattern for the two types of switching would support the assumption that the IFJ mediates switching between spe- cific contents (i.e., from one abstract rule to another or from one response mapping to another) via interactions with the relevant processing circuits for the newly relevant task set. That way, the IFJ initiates the updating of a task set by reconfiguring the functional brain network involved in the task.

\section{METHODS}

\section{Participants}

All 48 participants were native Germans of white ethnicity, without neurological or psychiatric history, and had normal or corrected-to-normal vision (24 women; age: $\mathrm{M}=$ 22.0 years, $S D=1.99$ years; 24 men, age: $M=22.6$ years, $S D=1.99$ years). The task-switching paradigm was administered in one fMRI session together with two other experiments that will be reported elsewhere. The study was approved by the local ethics committee of the Medical School of the University of Heidelberg, and participants gave written consent according to the Declaration of Helsinki.

\section{Behavioral Procedure}

Participants performed two different tasks on visually presented number stimuli depending on a task cue presented 300 msec before the number stimuli. The task cue remained on the screen until the end of the trial (see Figure 1), and participants were instructed to respond fast and accurately. A diamond cue indicated that participants

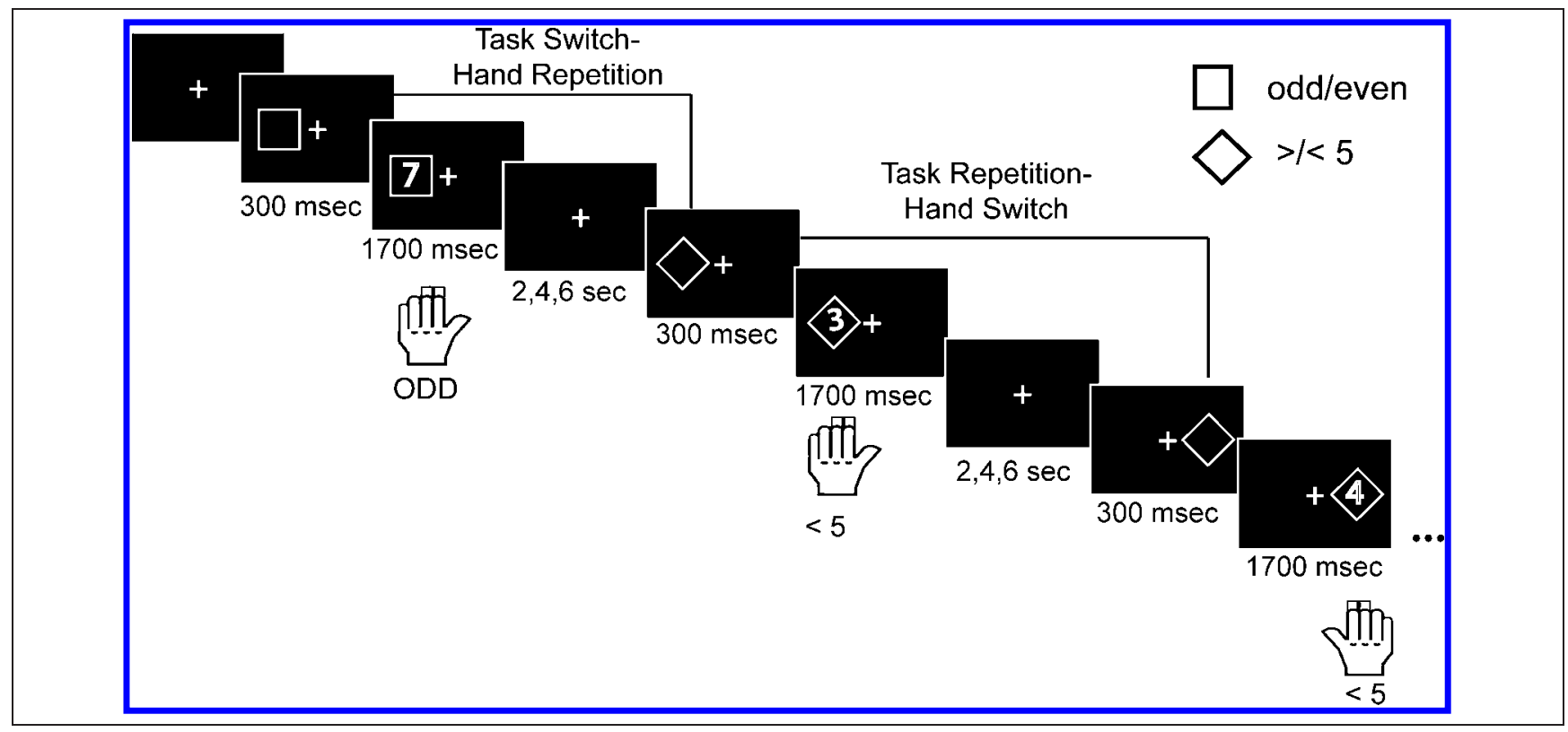

Figure 1. Task-switching paradigm. Depending on task cues (i.e., square vs. diamond), participants performed two different tasks on visually presented number stimuli (i.e., odd/even vs. smaller/larger five decisions). Participants responded with their left hand if cue and target were presented to the left of the fixation cross and with their right hand for presentation to the right of the fixation cross. Abstract rules and response hands were either repeated or switched from trial to trial, according to a pseudorandomly determined sequence. 
were to decide whether the number stimulus was smaller or larger than five. A square indicated to decide whether the number was odd or even. The number stimulus was presented in the center of the cue symbol for $1700 \mathrm{msec}$, followed by a variable intertrial interval of 2, 4, or $6 \mathrm{sec}$. In addition to the sequence of the two abstract rules ("tasks"), the location of the task cues was varied. Cues were presented either to the left or to the right of the fixation cross, indicating the response hand for the present trial. If cue and stimulus appeared to the left of the fixation cross, the response was to be given with the middle or index finger of the left hand, if it appeared to the right of the fixation cross, participants were to respond with the right middle or index finger. Participants responded with the left finger of the respective response hand if the stimulus was smaller than five or even and with the right finger if it was greater than five or odd. That way, four different types of trials emerged in a factorial $2 \times 2$ design: task repetition/hand repetition, task repetition/hand switch, task switch/hand repetition, and task switch/hand switch.

The task sequence was pseudorandomized with the restriction that not more than three trial type repetitions, two stimulus repetitions, and three response repetitions occurred in sequence. Transitions between conditions were balanced evenly. Note that the subjects were not informed about these restrictions. For the switching conditions of interest (task switch/hand repeat and task repetition/ hand switch), it is also unlikely that subjects built up expectancies (e.g., that a switch would occur after three task repetitions) on the basis of their experience with the task. This is because the different types of transitions of these higher order sequences (e.g., task types A-A-A-B) were extremely rare (about three to four trials per sequence in the whole experiment) and, most importantly, could not be predicted in full detail as three different types of switches (i.e., task switch, response switch, or both) could occur in Trial 4 of the episode. The experiment comprised two runs of approximately 9 min and $30 \mathrm{sec}$ length. Each run involved 96 trials, resulting in $47-48$ trials for each of the four conditions. Participants received two blocks of training with 64 trials each directly before the MRI session.

\section{fMRI Procedure}

All images were acquired with a 3-T Siemens Trio MRI scanner equipped with a fast gradient system for EPI. We used a birdcage head coil and stabilized participants with cushions to restrict head motion comfortably. Functional images were acquired in two runs, using a whole-brain one-shot gradient-echo, echo-planar sequence (echo time $=30 \mathrm{msec}$, matrix size $=64 \times 64$, field of view $=$ $24 \mathrm{~cm}$, flip angle $=83^{\circ}$, repetition time $=2 \mathrm{sec}$ ). Each functional volume consisted of 32 axial slices with 3-mm thickness and 1-mm interslice gap. We also acquired a structural T1-weighted 3-D MPRAGE scan at the end of the experiment (matrix size $=256 \times 256$, slice thickness $=$ $1.0 \mathrm{~mm}$, flip angle $=30^{\circ}$ ). In addition, we acquired a 2-D T1-weighted image using the same slice prescription as for functional scans. Anatomical images were used for the normalization of the functional data to the Montreal Neurological Institute (MNI) atlas space.

\section{fMRI Data Analyses}

All analyses were carried out with SPM5 (www.fil.ion.ucl.ac. $\mathrm{uk} / \mathrm{spm} / \mathrm{spm} 5 . \mathrm{html}$ ). First, each participant's functional data set was slice-time corrected, motion corrected, and unwarped and then coregistered to the anatomical data. After segmentation, the data were spatially normalized into the standard MNI atlas space. Data were then smoothed with an 8-mm FWHM Gaussian kernel and high-pass filtered $(128 \mathrm{sec})$ during statistical analysis. We applied the general linear model for serially autocorrelated data (Friston et al., 1995), included one covariate for each task condition in relation to the preceding trial. Importantly, to enable hand-specific functional connectivity analyses (see below), the four conditions were separated according to the respective response hands, resulting in eight predictor covariates in the general linear model: task repetition/hand repetition (1. left-left; 2. right-right); task repetition/hand switch (3. left-right; 4. right-left); task switch/hand repetition (5. left-left; 6. right-right); task switch/hand switch (7. left-right; 8. right-left). In addition, we included one covariate for error trials. To protect the whole-brain analysis against false positive activations, we used a double-threshold approach, that is, combining a voxel-based threshold with a minimum cluster size (Forman et al., 1995). This nonarbitrary voxel cluster size was determined on the basis of a Monte Carlo simulation (10,000 iterations) as implemented in AFNI's alphasim tool (Ward, 2000). With our brain volume and an individual voxel height threshold of $T>3.11$ ( $p<.001$, uncorrected), it could be determined that a cluster size of $k=66$ contiguous voxels ensured an overall image-wise false-positive rate of 5\%. Activations exceeding this double threshold are, therefore, considered to be activated at an experiment-wise threshold of $p<$ .05 , corrected for multiple comparisons. In addition to the contrasts for task switching (task switch/hand repetition vs. task repetition/hand repetition) and hand switching (hand switch/task repetition vs. hand repetition/task repetition), we also tested which regions were activated in the conjunction of both contrasts (Nichols, Brett, Andersson, Wager, \& Poline, 2005). Parameter estimates were extracted from a spherical $(8 \mathrm{~mm})$ mask surrounding the conjunction peaks to illustrate the activity pattern in these regions.

\section{Psychophysiological Interactions}

To investigate the functional connectivity of the IFJ region depending on the type of switching, we used the method of psychophysiological interactions (PPIs; Friston et al., 
1997). The aim of a PPI analysis is to explain neural responses in one brain region in terms of the interaction between the neural responses in another brain region and a specific psychological context.

We used the left IFJ spherical mask from the conjunction analysis as seed region and calculated the PPI term as the product of the mean deconvolved time course in this region (Gitelman, Penny, Ashburner, \& Friston, 2003) and the respective psychological variables (1. task switch/ hand repetition vs. task repetition/hand repetition and 2. hand switch/task repetition vs. hand repetition/task repetition). To separate connectivity patterns during hand switching with respect to the direction of the hand switch, we calculated additional PPIs with the psychological variables 3. hand switch left-right versus hand repetition right-right, and 4. hand switch right-left versus hand repetition left-left. For each psychological variable, the respective three variables (time course in seed region, psychological variable, and interaction term) were entered into a new general linear model, separately for each subject. For the PPI analyses, we applied a combined voxelwise threshold of $p<.005$ and cluster size threshold of $k>149$ voxels on the basis of the Monte Carlo simulation described above. That way we maintained the same corrected $p$ value of $p<.05$ as for the activation results but allowed for a more sensitive analysis with respect to voxelwise thresholds in the connectivity results.

\section{RESULTS}

\section{Behavioral Results}

Behavioral data were analyzed in a 2 (task repetition vs. switch) $\times 2$ (hand repetition vs. switch) repeated measures ANOVA. Response times were clearly modulated by the presence of a task switch, $F(1,47)=153.2, p<.001$. RTs were increased in task switch compared with task repetition trials (see Figure 2). There was no significant RT main effect for Hand Switching when collapsed across task switching and repetition conditions $(p=.25)$. The interaction pattern between the two factors, $F(1,47)=20.1$, $p<.001$, however, indicated that the hand-switching effects depended strongly on the presence or absence of a task switch. Hand-switching costs were present during task repetitions, $t(47)=3.8, p<.001$, whereas there was a benefit for hand switching in task-switching situations, $t(47)=2.2, p=.03$. Thus, subjects were faster in hand switching when they could also switch the tasks. On the basis of this interaction effect, we decided to analyze the switching effects in the fMRI data from the pure switching contrasts, that is, task switching versus repetition when the hand remained the same and hand switching versus repetition when the abstract task rule remained the same, respectively. Additional analyses of the effects of handswitching order (left to right vs. right to left) revealed an interaction effect with the hand switch versus repetition factor, $F(1,47)=6.66, p<.013)$. Although switching costs in the RTs for switching from right to left hand (compared with response repetitions on the left) were present, $t(47)=$ $3.74, p<.001$, these were strongly reduced for switches from the left to the dominant right hand, $t(47)=1.42$, $p=.16$.

Error rates were higher for task switches compared with task repetitions, $F(1,47)=19.3, p<.001$, although there was no main effect for switching hands. Numerically, there was a similar interaction pattern as in the RTs, however, not significant ( $p=.17$; cf. Figure 2$)$. The direction of the response hand switch (left to right hand vs. right to left hand) did not affect error rates, $F(1,47)=0.01, p=.93$.

\section{fMRI Results}

\section{Task Switching}

Switching of abstract task rules, as isolated on the basis of the comparison of task switch/hand repetition and task repetition/hand repetition trials, revealed activity changes in a network previously shown to be relevant for task switching (Table 1). This network included a large cluster along the left posterior inferior frontal sulcus (IFS) including the IFJ (Figure 3, green color code). In addition, task switching was associated with activity in the left superior frontal sulcus and the left intraparietal sulcus and precuneus.
Figure 2. Behavioral results. Effects of switching condition on RTs (left) and error rates (right). Error bars: SEM.

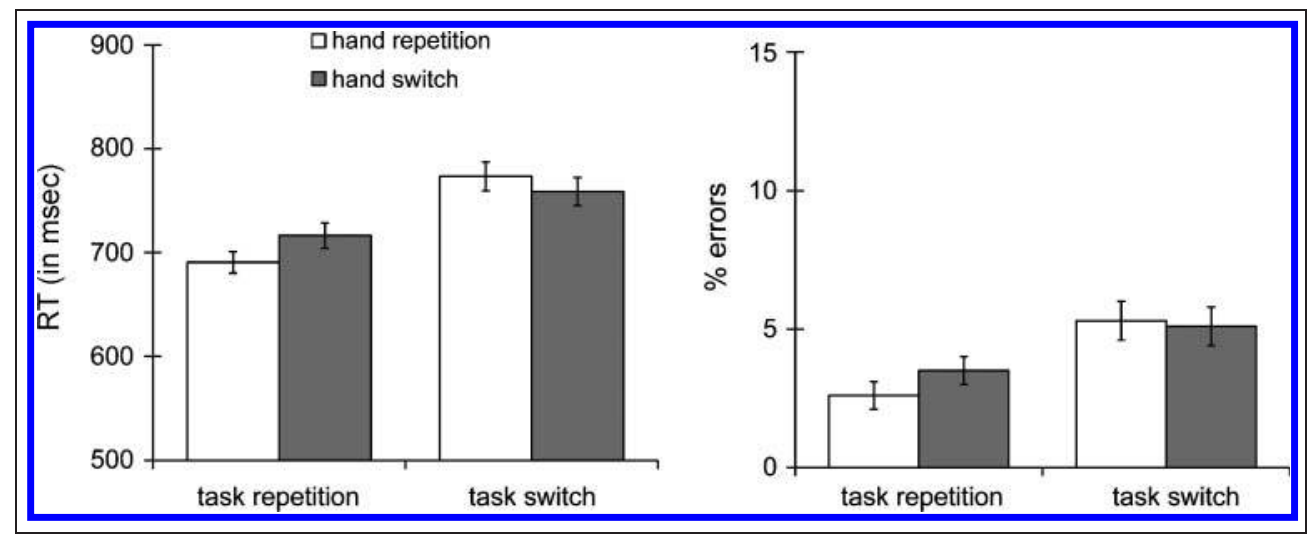


Table 1. Anatomical Location and MNI Coordinates for Abstract Rule Switch versus Repetition Contrast, Hand Switch versus Hand Repetition Contrast, and the Conjunction of Both $(p<.001, k>66$ voxels $)$

\begin{tabular}{|c|c|c|c|c|c|c|c|}
\hline \multirow[b]{2}{*}{ Region } & \multirow[b]{2}{*}{ Hem } & \multirow[b]{2}{*}{$B A$} & \multicolumn{3}{|c|}{ MNI Coordinates } & \multirow[b]{2}{*}{$t$} & \multirow[b]{2}{*}{ Cluster Size } \\
\hline & & & $x$ & $y$ & $z$ & & \\
\hline \multicolumn{8}{|l|}{ Abstract rule switching } \\
\hline IFS & $\mathrm{L}$ & 46 & -50 & 12 & 22 & 4.16 & 187 \\
\hline IFJ & $\mathrm{L}$ & $9 / 6$ & -52 & 6 & 38 & 3.78 & included \\
\hline Superior frontal sulcus & $\mathrm{L}$ & 6 & -26 & -4 & 54 & 3.74 & 77 \\
\hline Precuneus & $\mathrm{L}$ & 7 & -12 & -68 & 54 & 4.26 & 1016 \\
\hline Intraparietal sulcus & $\mathrm{L}$ & 40 & -32 & -68 & 48 & 4.10 & included \\
\hline \multicolumn{8}{|l|}{ Response hand switching } \\
\hline IFJ & $\mathrm{L}$ & $9 / 6$ & -52 & 0 & 38 & 4.91 & 251 \\
\hline Superior frontal sulcus & $\mathrm{L}$ & 6 & -22 & -4 & 50 & 5.94 & 1500 \\
\hline Intraparietal sulcus & $\mathrm{L}$ & 40 & -44 & -26 & 40 & 4.69 & 512 \\
\hline Postcentral sulcus & $\mathrm{R}$ & $3 / 4$ & 36 & -32 & 40 & 5.57 & 1684 \\
\hline Precuneus/posterior cingulate & $\mathrm{L} / \mathrm{R}$ & $7 / 31$ & 12 & -26 & 16 & 5.40 & 4186 \\
\hline Precuneus & $\mathrm{R}$ & 7 & 12 & -58 & 62 & 4.27 & 203 \\
\hline Angular gyrus & $\mathrm{L}$ & 39 & -44 & -76 & 28 & 4.31 & 207 \\
\hline Thalamus & $\mathrm{R}$ & & 16 & -22 & 8 & 4.55 & 346 \\
\hline Caudate nucleus & $\mathrm{R}$ & & 20 & 2 & 20 & 3.80 & 78 \\
\hline Hippocampus & $\mathrm{L}$ & & -22 & -8 & -26 & 4.22 & 74 \\
\hline Cerebellum & $\mathrm{R}$ & & 30 & -50 & -32 & 4.70 & 700 \\
\hline Cerebellum & $\mathrm{R}$ & & 2 & -54 & -10 & 4.44 & 192 \\
\hline Cerebellum & $\mathrm{L}$ & & -26 & -30 & -28 & 4.17 & 115 \\
\hline \multicolumn{8}{|l|}{ Conjunction } \\
\hline IFJ & $\mathrm{L}$ & $9 / 6$ & -52 & 4 & 38 & 3.73 & 76 \\
\hline Superior parietal lobe/precuneus & $\mathrm{L}$ & 7 & -14 & -68 & 56 & 4.26 & 316 \\
\hline Superior frontal sulcus & $\mathrm{L}$ & 6 & -26 & -4 & 54 & 3.74 & 71 \\
\hline
\end{tabular}

Hem $=$ hemisphere; $\mathrm{BA}=$ Brodmann's area $\mathrm{L}=$ left $\mathrm{R}=$ right.

\section{Response Hand Switching}

Performing the same task but having to switch the response hand (hand switch/task repetition vs. hand repetition/task repetition) was also associated with activity changes in the left IFJ region (Figure 3, gray color code). In addition, response switching was associated with activity in the left superior frontal sulcus, parietal regions including the left intraparietal sulcus, the right postcentral sulcus, and the precuneus bilaterally, and also in the left angular gyrus, the right thalamus, the right caudate nucleus, the left hippocampus and the cerebellum bilaterally (Table 1) -all regions showing increased activity during response switching compared with response repetitions. Overall, hand switching clearly showed a more extended network of activated regions compared with abstract task rule switching, at the same statistical level of significance (Table 1).

\section{Conjunction Analysis}

Three regions showed overlapping activity for task switching and response hand switching. This included the left IFJ (Figure 3, red color code), a region in the left superior frontal sulcus, and one activity peak in the left superior parietal cortex (Table 1). The analysis of the ROI data from the overlapping IFJ region indicated that, compared with the task repeat/hand repeat condition, the signal increase in the IFJ was equally strong for abstract rule switches as for hand switches, that is, there was no difference in IFJ 


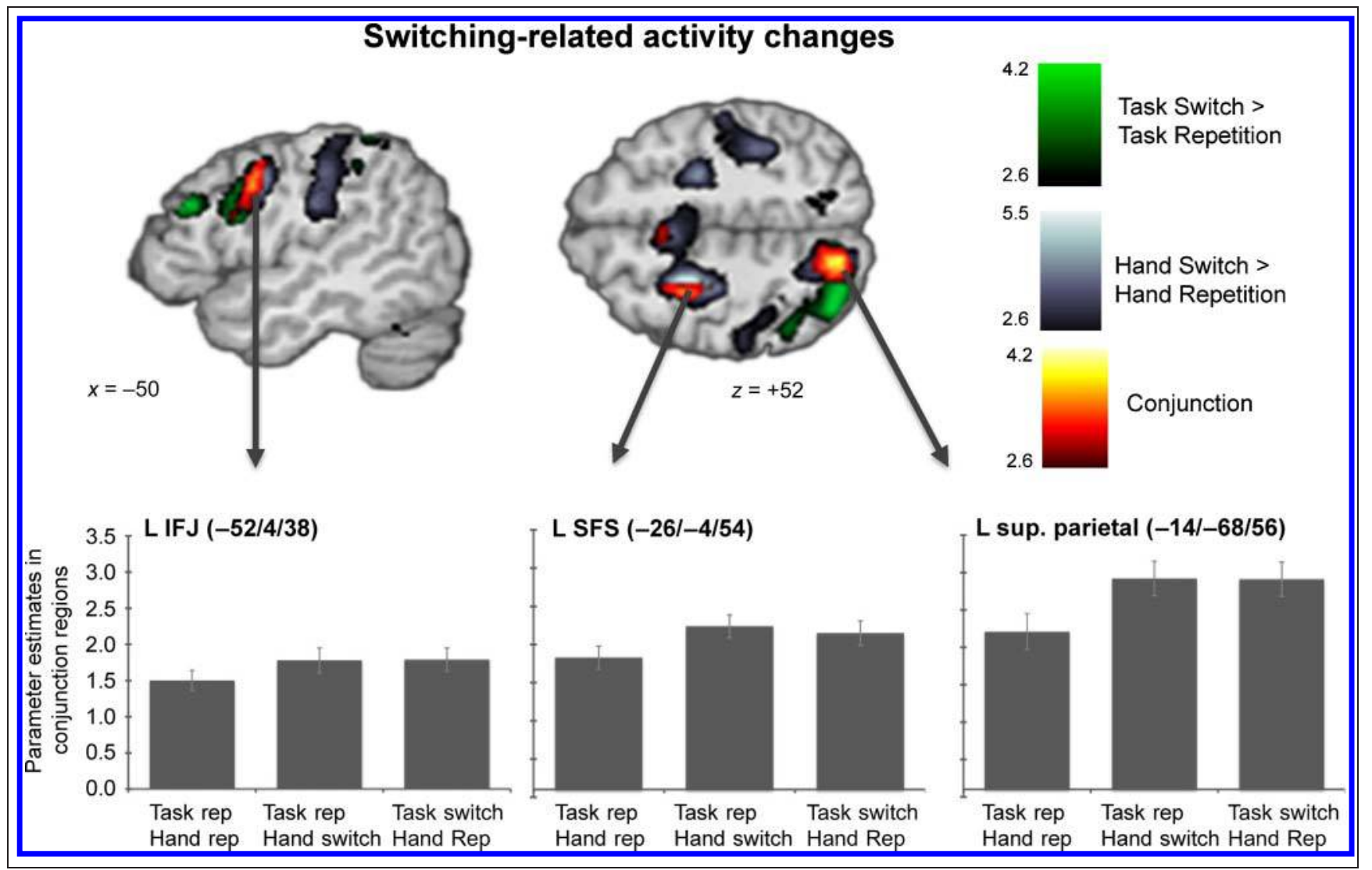

Figure 3. Activity maps for abstract rule switching (task switch/hand repetition > task repetition/hand repetition; green color code), hand switching (hand switch/task repetition $>$ hand repetition/task repetition; gray color code), and the conjunction of both (red color code, all shown at $p<.005)$. Parameter estimates are presented below to illustrate the effects in those regions jointly involved in abstract rule switch and hand switching. Error bars: SEM.

activation strength between task- and hand-switching costs, $t(47)=.04, p=.96$ (see Figure 3). Neither was there any difference in activation observed in the superior frontal sulcus, $t(47)=1.04, p=.31$, or the superior parietal cortex, $t(47)=-.08, p=.94$. Also, IFJ activity changes for both contents of the switch were highly correlated (Figure 4)-individuals who showed strong activity related to task switching also showed strong activity related to hand switching in the IFJ $(r=.35, p=.015)$.

\section{Switching-specific Functional Connectivity (PPI)}

The overlapping region in the IFJ was further analyzed in terms of task-specific connectivity patterns. For abstract rule switches compared with repetitions (i.e., when the response hand remained the same but the abstract rule changed), there was increased functional connectivity of the IFJ with the anterior PFC in the banks of the left IFS, with the left superior frontal sulcus, the left parahippocampal gyrus and hippocampus, the right inferior temporal gyrus, and the left occipital cortex (Figure 5A; Table 2). This connectivity pattern was specific for task switching and not present for hand switching as can be seen in the bar plots in Figure 5A. Specific functional connectivity of the IFJ during hand switching (i.e., when the task remained the same) was only present when PPIs were analyzed separately depending on the direction of the hand switch (i.e., from left to right vs. right-right and from right to left vs. left-left). Only in the comparison of hand switches from

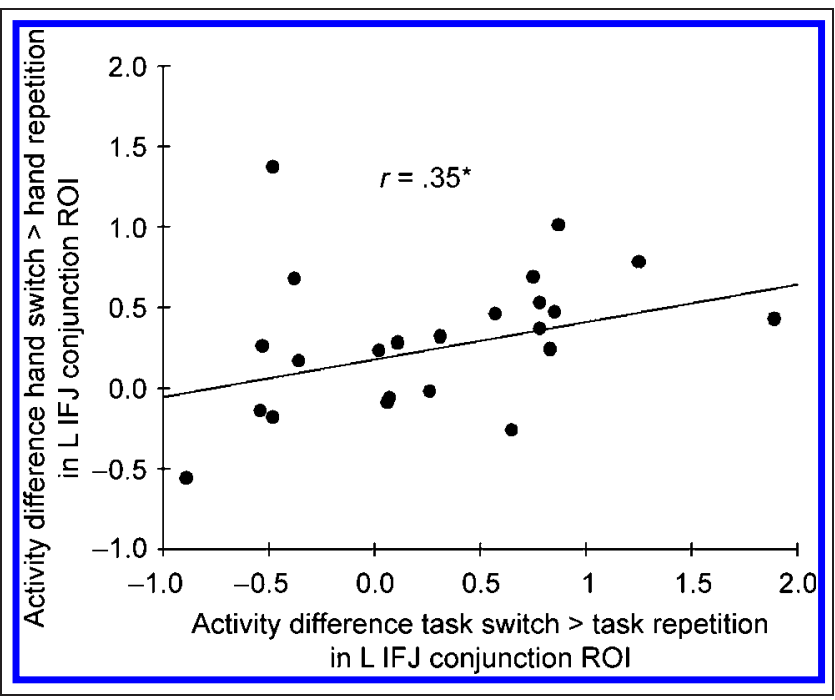

Figure 4. Correlation of abstract rule switching effects and hand-switching effects in IFJ conjunction region (parameter estimates; * $p<.05$ ). 
left to right hand, as compared with hand repetitions with the right hand, was there an increased functional coupling of the left IFJ with the left central sulcus as well as postcentral regions including the intraparietal sulcus (Figure 5B). To investigate whether homologous regions in the right hemisphere might be connected to the right IFJ for switches in the other direction (i.e., right to left vs. leftleft repetitions) we used a homologous right IFJ seed region (8-mm sphere at MNI coordinates: $x=52, y=4$, $z=38$ ) and performed an additional PPI analysis. The results indicate that no comparable effect of response switch versus repetition on functional connectivity was present for switches from the right hand to the left hand.

\section{DISCUSSION}

In the present study, we compared the overlap in activity and functional connectivity for switching between two dif- ferent elements of a task set - the abstract task rules guiding stimulus categorization and the specific response set as defined via the response hand. Our data confirm a rather general involvement of the IFJ region in both types of switching. Activity changes compared with a condition where the task and the response hand were repeated were nearly identical for both types of switching, and the extent of activity change in both switching conditions was correlated within individuals. The functional connectivity data, however, showed a highly distinct pattern of task-specific connectivity for each type of switching.

\section{Abstract Rule Switching}

During switching between abstract rules, IFJ activity was specifically correlated with activity in the left anterior PFC. Note that, although no suprathreshold activity was present during task switching in this exact anterior prefrontal
Figure 5. Functional connectivity (PPI) with left IFJ conjunction region as seed region, separately for psychological factors (A) task switch/hand repetition $>$ task repetition/hand repetition and (B) hand switch/task repetition $>$ hand repetition/ task repetition, respectively $(p<.005)$. Bar graphs illustrate effects for both psychological factors to show the selectivity of the obtained results. Error bars: SEM.

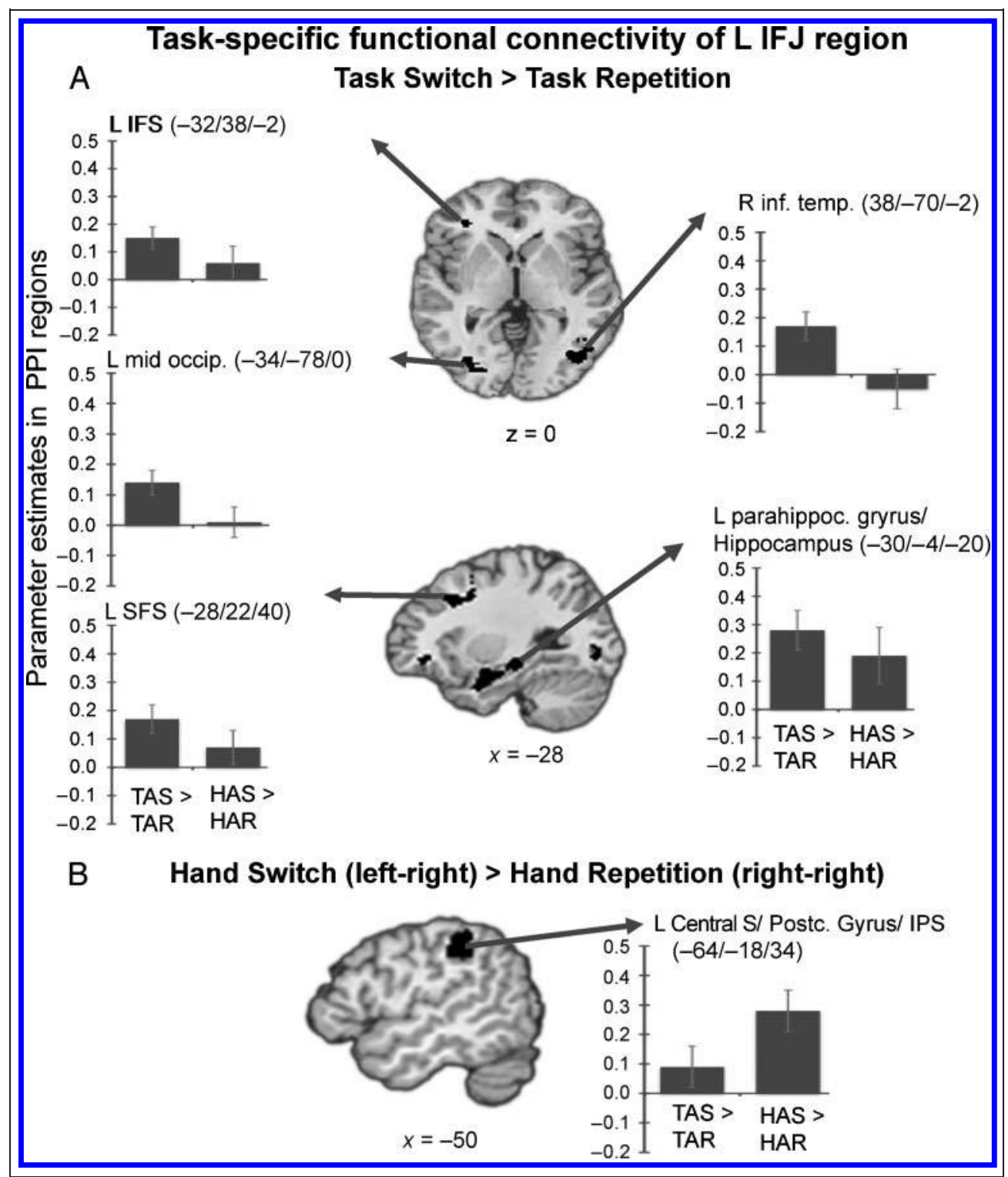


Table 2. Anatomical Location and MNI Coordinates of Functional Connectivity Analysis (PPI) with Left IFJ Conjunction Region as Seed Region, Separately for Abstract Rule Switch versus Repetition and for Hand Switch (Left to Right) versus Hand Repetition (Right-Right; $p<.005, k>149$ )

\begin{tabular}{|c|c|c|c|c|c|c|c|}
\hline \multirow[b]{2}{*}{ Region } & \multirow[b]{2}{*}{ Hem } & \multirow[b]{2}{*}{$B A$} & \multicolumn{3}{|c|}{ MNI Coordinates } & \multirow[b]{2}{*}{$t$} & \multirow[b]{2}{*}{ Cluster Size } \\
\hline & & & $x$ & $y$ & $z$ & & \\
\hline \multicolumn{8}{|l|}{ PPI abstract rule switching } \\
\hline IFS & $\mathrm{L}$ & $11 / 47$ & -32 & 38 & -2 & 4.03 & 158 \\
\hline Superior frontal sulcus & $\mathrm{L}$ & $6 / 9$ & -28 & 22 & 40 & 3.86 & 181 \\
\hline Parahippocampal gyrus & $\mathrm{L}$ & & -30 & -4 & -20 & 4.61 & 421 \\
\hline Hippocampus & $\mathrm{L}$ & & -30 & -26 & -10 & 4.00 & included \\
\hline Inferior temporal gyrus & $\mathrm{R}$ & 37 & 38 & -70 & -2 & 3.85 & 205 \\
\hline Middle occipital gyrus & $\mathrm{L}$ & 18 & -34 & -78 & 0 & 3.87 & 157 \\
\hline \multicolumn{8}{|c|}{ PPI response hand switching (left-right vs. right-right) } \\
\hline Central sulcus & $\mathrm{L}$ & $3 / 4$ & -64 & -18 & 34 & 4.14 & 574 \\
\hline Postcentral gyrus/intraparietal sulcus & $\mathrm{L}$ & 40 & -48 & -36 & 44 & 4.01 & included \\
\hline
\end{tabular}

Hem $=$ Hemisphere, $\mathrm{BA}=$ Brodmann's area; $\mathrm{L}=$ left; $\mathrm{R}=$ right.

region identified in the PPI analysis, a post hoc analysis of the activation data with a lower threshold of $p<.005$, uncorrected for cluster size, revealed a small cluster related to task switching in the left anterior PFC $(x=-48, y=$ $44 ; z=8 ; k=13 ; t_{\max }=2.73$ ) adjacent to the region found to be activated when the contrast for abstract rule switching was pooled across response hand repetitions and switches (Stelzel, Basten, Montag, Reuter, \& Fiebach, 2010). Endogenous task preparation has previously been associated with activity in anterior lateral prefrontal regions (Forstmann, Brass, Koch, \& von Cramon, 2005; Rowe et al., 2005) and might underlie the present pattern of connectivity. Specifically, Forstmann et al. (2005) were able to show that anterior portions of PFC are associated with the endogenous preparation for a task switch when the switch cue does not provide the complete information about the upcoming tasks rule. Our finding of a lateral frontal network for cue-based switching between abstract task representations indicates that subjects might partly rely on endogenous representations even when relying on the task-specifying cue is sufficient for successful task performance. Specifically, they might use a changing task cue as a signal that the task is changing and then support cue-based updating of the task representation with endogenous processes. Such a strategy would also be consistent with hierarchical conceptions of lateral frontal organization (Badre, 2008; Fuster, 2006; Koechlin, Ody, \& Kouneiher, 2003), where it is assumed that more abstract, goal-related processing is related to more anterior prefrontal regions. Whereas these models assume that the abstractness of processing defines the posterior-to-anterior gradient in the lateral frontal cortex, other models focus on the abstractness of representation as the defining dimension of PFC recruitment (Wood
\& Grafman, 2003). This is a crucial distinction with respect to the functionality of the lateral frontal cortex in rule processing. Are rules and intentions represented and maintained in this most anterior region of the cortex or are signals from there sent to more posterior regions to bias processing in regions that actually represent the task-relevant information? Although the role of the IFJ is usually seen as related to a specific process, namely the updating of task representations (Stelzel et al., 2010; Brass et al., 2005; Brass \& von Cramon, 2002), some recent evidence from multivoxel pattern analysis suggests that activity in anterior PFC can be associated with the representation of abstract rules (Bode \& Haynes, 2009; Haynes et al., 2007). Whether anterior prefrontal activity can be consistently decoded as being related to the representation of specific task rules-that is, whether abstract rules and goals are actually stored in the anterior PFC—is still under debate (Sakai, 2008).

With respect to the anterior prefrontal connectivity with the IFJ observed during switching between abstract rules, one alternative hypothesis to an endogenous switching process (Forstmann et al., 2005) could be that endogenous preparation for a switch might lead to stronger representations in anterior PFC than cue-based switches, thus involving regions presumably associated with the representation of abstract rules to a higher degree. The interaction between a region that sends an updating signal (i.e., IFJ) and a region that is related to the representation of an abstract rule (i.e., anterior PFC) provides a comprehensible interpretation for the present functional connectivity findings.

Additional switching-related connectivity of the IFJ with the left superior frontal sulcus might be associated with spatial rehearsal processes involved in implementing the new 
task rule (Curtis, Cole, Rao, \& D'Esposito, 2005), for example, related to imagining the upcoming rule on a number line. Inferior temporal and midoccipital increases in connectivity might reflect increased top-down attention on certain stimulus attributes when tasks need to be switched (Desimone \& Duncan, 1995).

In addition, increased functional connectivity of the IFJ with the hippocampus and parahippocampal gyrus was present during task switching. This fronto-hippocampal connectivity, however, was rather surprising, as (univariate) hippocampal activation during task switching was so far only shown for task switching in predictable sequences (Dreher, Koechlin, Ali, \& Grafman, 2002), whereas a pseudorandomized task order was applied in the present study. We speculate that the switching-related changes in functional connectivity might reflect increased effort in the collaboration of the lateral frontal cortex with the hippocampus for the cue-based retrieval of a newly relevant task rule in taskswitching situations, although hippocampal activity per se is not strong enough to pass the statistical threshold. Again, when using an uncorrected threshold of $p<.005$, a smaller cluster of 29 voxels was present in this region $(x=-18, y=$ $-24 ; z=-6 ; t_{\max }=3.12$ ). Taken together, the connectivity results support the generally assumed role of the left IFJ as a central hub in updating task representation by orchestrating various task-related regions when a new abstract task rule needs to be implemented.

\section{Response Hand Switching}

For hand switching, functional connectivity was generally less strong, although activity related to response hand switching was much more widespread and overall stronger than for task switching. Only when analyzing hand switches separately with respect to the direction of the switch, there was a selective increase in connectivity between left IFJ and left motor cortex and motor-related parietal regions (Culham \& Kanwisher, 2001) for switching from left to right. Although this effect indicates a switching-related interaction of these regions and fits nicely to previous reports on interactions with motor regions during action selection (Rowe et al., 2005), the asymmetry of this effect is less clear. It might be that stronger functional connectivity with left motor cortex for right hand responses is needed when switching away from the nondominant left hand as this condition needed more control in the first place that might persist through the next trial. Alternatively, right-hand repetitions might be characterized by more pronounced decreases in functional coupling and, thus, lead to this differential effect. The RT data, showing identical hand repetition RTs for left- and right-hand repetitions $(t(47)=$ $0.22, p=.82$ ), seem to support the first view.

Importantly, the obtained selective connectivity effects with the motor cortex during response switching support the notion that the dominant switch dimension in situations where the task cue switched location was indeed the response representation and not just a switch in atten- tional focus. Previous evidence suggests that the superior parietal cortex is a common locus for switching attention and switching between abstract task rules (Chiu \& Yantis, 2009). We provide further evidence for this, showing common activity for response and abstract rule switching in parietal and superior frontal regions, that is, regions commonly related to changes in spatial attention (Desimone \& Duncan, 1995). However, the present data complement these previous findings, indicating that switching between motor responses and abstract rule switching additionally share computational demands in the left IFJ. An experiment manipulating both attentional switching and response hand switching independently might further elucidate these interpretations. Taken together, the results of Chiu and Yantis (2009) and our results indicate that switching between different aspects of a task, such as stimulus location, abstract task rule, and response hand overlap to different degrees-with the IFJ being particularly relevant for switching between abstract task rules and response representations.

\section{Cognitive Implications}

In cognitive terms, the present findings support the view that the reconfiguration of a task set (Meiran, 1996; Monsell, 1996) is a controlled process that requires the updating of the previously relevant task elements. From the experimental task-switching literature, it is well known that this reconfiguration is hardest when there is high overlap between the elements (i.e., stimuli, abstract rules, and/ or responses) of the two task sets (Hommel, Müsseler, Aschersleben, \& Prinz, 2002; Logan \& Gordon, 2001). The present imaging results suggest that, computationally, reconfiguring an abstract rule or the relevant response set might involve similar signaling cascades, with the IFJ being a common node for these different reconfigurations. Studies focusing on the processing in posterior task-relevant regions during task preparation (Wylie, Javitt, \& Foxe, 2006; Yeung, Nystrom, Aronson, \& Cohen, 2006) suggest that persisting activity from a previous task set might actually account for the additional effort involved in performing a subsequent task. Accordingly, resolving a compound of stimulus set, abstract rule set, and response set by refocusing attention can be considered as a prerequisite for the ability to perform two similar but not identical tasks in short succession (Hommel et al., 2002).

Switching effects shown in the present study further converge nicely with previous behavioral (Reuter, Philipp, Koch, \& Kathmann, 2006; Schuch \& Koch, 2004; Hommel et al., 2002; Meiran, 2000) and neurophysiological findings (Brass et al., 2003; Asaad, Rainer, \& Miller, 2000), suggesting that responses are coded in relation to their meaning, that is, the task they are associated with, thus leading to costs when the meaning has to be recoded. Previous work showed that response overlap between tasks leads to additional computational effort in the PFC during task switching compared with univalent response mappings where each 
task is associated with different response sets (Brass et al., 2003). Response mappings were generally bivalent in the present study, that is, all four response keys were associated with both tasks. In the case of task switching (with response hand repetitions), the same response hand has to be recoded with a different abstract rule. In the case of response hand switching (with task repetitions), in contrast, the response meaning needs to be resolved to be able to associate a different response hand with the same abstract rule. Although the IFJ seems to be involved in both types of recoding mechanisms, the most obvious difference was present in task-specific connectivities with anterior prefrontal and hippocampal as compared with motor regions, indicating that recoding is realized at different levels of abstraction.

An additional source of variability in sequential task performance relates to repetitions versus switches at the level of the specific response finger. It has been shown that even in trials where the response hand is repeated, task-switching costs are strongly increased when the response to the subsequent task is to be given with the exact same response finger, the so-called "reversed repetition" effect (Crone, Bunge, Van Der Molen, \& Ridderinkhof, 2006; Meiran \& Gotler, 2001; Kleinsorge \& Heuer, 1999). Although a post hoc analysis of the RT data for our hand repetition trials replicate this effect (interaction of task switch vs. repetition and response finger switch vs. repetition, $F(1,47)=9.11, p<.01)$ the number of trials with this additional factor was too low to be able to analyze the according fMRI data. Future studies might address the question of whether this recoding mechanism also involves the IFJ in interaction with a more restricted motor region.

In summary, this study further supports a general role of the posterior lateral frontal cortex, most specifically, the IFJ area, in updating task representations depending on the specific context of the required switch. Transient functional interactions with the anterior PFC and the hippocampus, however, separate abstract rule switching from response hand switching, which, in turn, is characterized by correlated activity in a motor network.

Reprint requests should be sent to Christine Stelzel, Division of Mind and Brain Research, Department of Psychiatry and Psychotherapy, Charité Universitätsmedizin Berlin, Charité Campus Mitte, Charitéplatz 1, D-10117 Berlin, Germany, or via e-mail: christine.stelzel@charite.de.

\section{REFERENCES}

Asaad, W. F., Rainer, G., \& Miller, E. K. (2000). Task-specific neural activity in the primate prefrontal cortex. Journal of Neurophysiology, 84, 451-459.

Badre, D. (2008). Cognitive control, hierarchy, and the rostro-caudal organization of the frontal lobes. Trends in Cognitive Sciences, 12, 193-200.

Barbas, H., \& Mesulam, M. (1985). Cortical afferent input to the principalis region of the rhesus monkey. Neuroscience, 15, 619-637.
Bode, S., \& Haynes, J.-D. (2009). Decoding sequential stages of task preparation in the human brain. Neuroimage, 45 , 606-613.

Brass, M., Derrfuss, J., Forstmann, B., \& von Cramon, D. Y. (2005). The role of the inferior frontal junction area in cognitive control. Trends in Cognitive Sciences, 9, 314-316.

Brass, M., Ruge, H., Meiran, N., Rubin, O., Koch, I., Zysset, S., et al. (2003). When the same response has different meanings: Recoding the response meaning in the lateral prefrontal cortex. Neuroimage, 20, 1026-1031.

Brass, M., \& von Cramon, D. Y. (2002). The role of the frontal cortex in task preparation. Cerebral Cortex, 12, 908-914.

Braver, T. S., Reynolds, J. R., \& Donaldson, D. I. (2003). Neural mechanisms of transient and sustained cognitive control during task switching. Neuron, 39, 713-726.

Chiu, Y. C., \& Yantis, S. (2009). A domain-independent source of cognitive control for task sets: Shifting spatial attention and switching categorization rules. Journal of Neuroscience, 29, 3930-3938.

Crone, E. A., Bunge, S. A., Van Der Molen, M. W., \& Ridderinkhof, K. R. (2006). Switching between tasks and responses: A developmental study. Developmental Science, 9, 278-287.

Culham, J. C., \& Kanwisher, N. G. (2001). Neuroimaging of cognitive functions in human parietal cortex. Current Opinion in Neurobiology, 11, 157-163.

Curtis, C. E., Cole, M. W., Rao, V. Y., \& D'Esposito, M. (2005). Canceling planned action: An FMRI study of countermanding saccades. Cerebral Cortex, 15, 1281-1289.

Derrfuss, J., Brass, M., Neumann, J., \& von Cramon, D. Y. (2005). Involvement of the inferior frontal junction in cognitive control: Meta-analyses of switching and Stroop studies. Human Brain Mapping, 25, 22-34.

Derrfuss, J., Brass, M., \& von Cramon, D. Y. (2004). Cognitive control in the posterior frontolateral cortex: Evidence from common activations in task coordination, interference control, and working memory. Neuroimage, 23, 604-612.

Desimone, R., \& Duncan, J. (1995). Neural mechanisms of selective visual attention. Annual Review of Neuroscience, 18, 193-222.

Dove, A., Pollmann, S., Schubert, T., Wiggins, C. J., \& von Cramon, D. Y. (2000). Prefrontal cortex activation in task switching: An event-related fMRI study. Brain Research. Cognitive Brain Research, 9, 103-109.

Dreher, J. C., Koechlin, E., Ali, S. O., \& Grafman, J. (2002). The roles of timing and task order during task switching. Neuroimage, 17, 95-109.

Forman, S. D., Cohen, J. D., Fitzgerald, M., Eddy, W. F., Mintun, M. A., \& Noll, D. C. (1995). Improved assessment of significant activation in functional magnetic resonance imaging (fMRI): Use of a cluster-size threshold. Magnetic Resonance in Medicine, 33, 636-647.

Forstmann, B. U., Brass, M., Koch, I., \& von Cramon, D. Y. (2005). Internally generated and directly cued task sets: An investigation with fMRI. Neuropsvchologia, 43, 943-952.

Forstmann, B. U., Brass, M., Koch, I., \& von Cramon, D. Y. (2006). Voluntary selection of task sets revealed by functional magnetic resonance imaging. Journal of Cognitive Neuroscience, 18, 388-398.

Friston, K., Holmes, A., Worsley, K., Poline, J., Frith, C., \& Frackowiak, R. (1995). Statistical parametric maps in functional imaging: A general linear approach. Human Brain Mapping, 2, 189-210.

Friston, K. J., Buechel, C., Fink, G. R., Morris, J., Rolls, E., \& Dolan, R. J. (1997). Psychophysiological and modulatory interactions in neuroimaging. Neuroimage, 6, 218-229. 
Fuster, J. M. (2006). The cognit: A network model of cortical representation. International Iournal of Psychophysiology, 60, 125-132.

Gitelman, D. R., Penny, W. D., Ashburner, J., \& Friston, K. J. (2003). Modeling regional and psychophysiologic interactions in fMRI: The importance of hemodynamic deconvolution. Neuroimage, 19, 200-207.

Haynes, J.-D., Sakai, K., Rees, G., Gilbert, S., Frith, C., \& Passingham, R. E. (2007). Reading hidden intentions in the human brain. Current Biology, 17, 323-328.

Hommel, B., Müsseler, J., Aschersleben, G., \& Prinz, W. (2002). The theory of event coding (TEC): A framework for perception and action planning. Behavioral and Brain Sciences, 24, 849-878.

Kleinsorge, T., \& Heuer, H. (1999). Hierarchical switching in a multidimensional task space. Psychological Research. 62, 300-312.

Koechlin, E., Ody, C., \& Kouneiher, F. (2003). The architecture of cognitive control in the human prefrontal cortex. Science, 302, 1181-1185.

Logan, G. D., \& Gordon, R. D. (2001). Executive control of visual attention in dual-task situations. Psychological Review, 108, 393-434.

Meiran, N. (1996). Reconfiguration of processing mode prior to task performance. Journal of Experimental Psychology Learning Memory and Cognition, 22, 1423-1442.

Meiran, N. (2000). Reconfiguration of stimulus task sets and response task sets during task switching. In S. Monsell \& J. Driver (Eds.), Control of cognitive processes: Attention and performance XVIII (pp. 377-400). Cambridge, MA: MIT Press.

Meiran, N., \& Gotler, A. (2001). Modelling cognitive control in task switching and ageing. European Journal of Cognitive Psychology, 13, 165-186.

Monsell, S. (1996). Control of mental processes. In V. Bruce (Ed.), Unsolved mysteries of the mind: Tutorial essays in cognition (pp. 93-148). London: Erlbaum (UK) Taylor \& Francis.

Nichols, T., Brett, M., Andersson, J., Wager, T., \& Poline, J. B. (2005). Valid conjunction inference with the minimum statistic. Neuroimage, 25, 653-660.

Pandya, D., \& Barnes, C. (1987). Architecture and connections of the frontal lobe. In E. Perecman (Ed.), The frontal lobes revisited (pp. 41-72). New York: The IRBN Press.
Reuter, B., Philipp, A. M., Koch, I., \& Kathmann, N. (2006). Effects of switching between leftward and rightward pro- and antisaccades. Biological Psychology, 72, 88-95.

Rogers, R. D., \& Monsell, S. (1995). Costs of a predictible switch between simple cognitive tasks. Lournal of Experimental Psychologv: General, 124, 207-231.

Rowe, J. B., Stephan, K. E., Friston, K., Frackowiak, R. S. J., \& Passingham, R. E. (2005). The prefrontal cortex shows context-specific changes in effective connectivity to motor or visual cortex during the selection of action or colour. Cerebral Cortex, 15, 85-95.

Sakai, K. (2008). Task set and prefrontal cortex. Anmual Review of Neuroscience, 31, 219-245.

Schuch, S., \& Koch, I. (2003). The role of response selection for inhibition of task sets in task shifting. Journal of Experimental Psvchologv: Human Perception and Performance, 29, 92-105.

Schuch, S., \& Koch, I. (2004). The costs of changing the representation of action: Response repetition and response-response compatibility in dual tasks. Journal of Experimental Psychology: Human Perception and Performance, 30, 566-582.

Stelzel, C., Basten, U., Montag, C., Reuter, M., \& Fiebach, C. J. (2010). Frontostriatal involvement in task switching depends on genetic differences in $\mathrm{d} 2$ receptor density. Lournal of Neuroscience, 30, 14205-14212.

Stephan, K. E., Marshall, J. C., Friston, K. J., Rowe, J. B., Ritzl, A., Zilles, K., et al. (2003). Lateralized cognitive processes and lateralized task control in the human brain. Science, 301, 384-386.

Ward, B. (2000). Simultaneous inference for fMRI data. AFNI $3 \mathrm{dDeconvolve}$ Documentation, Medical College of Wisconsin. Retrieved August 2007 from http://afni.nimh.nih.gov/afni/ docpdf/AlphaSim.pdf.

Wood, J. N., \& Grafman, J. (2003). Human prefrontal cortex: Processing and representational perspectives. Nature Reviews Neuroscience, 4, 139-147.

Wylie, G. R., Javitt, D. C., \& Foxe, J. J. (2006). Jumping the gun: Is effective preparation contingent upon anticipatory activation in task-relevant neural circuitry? Cerebral Cortex, 16, 394-404.

Yeung, N., Nystrom, L. E., Aronson, J. A., \& Cohen, J. D. (2006). Between-task competition and cognitive control in task switching. Journal of Neuroscience, 26, 1429-1438. 


\section{This article has been cited by:}

1. Paul S. Muhle-Karbe, Wouter De Baene, Marcel Brass. 2014. Do tasks matter in task switching? Dissociating domain-general from context-specific brain activity. NeuroImage 99, 332-341. [CrossRef]

2. Wouter De Baene, Marcel Brass. 2014. Dissociating strategy-dependent and independent components in task preparation. Neuropsychologia 62, 331-340. [CrossRef]

3. Christine Stelzel, Christian J. Fiebach, Roshan Cools, Sharwin Tafazoli, Mark D'Esposito. 2013. Dissociable fronto-striatal effects of dopamine D2 receptor stimulation on cognitive versus motor flexibility. Cortex 49, 2799-2811. [CrossRef]

4. Deborah J. Serrien, Michiel M. Sovijärvi-Spapé. 2013. Cognitive control of response inhibition and switching: Hemispheric lateralization and hand preference. Brain and Cognition 82, 283-290. [CrossRef]

5. Diana J. N. Armbruster, Kai Ueltzhöffer, Ulrike Basten, Christian J. Fiebach. 2012. Prefrontal Cortical Mechanisms Underlying Individual Differences in Cognitive Flexibility and Stability. Journal of Cognitive Neuroscience 24:12, 2385-2399. [Abstract] [Full Text] [PDF] [PDF Plus]

6. Wouter De Baene, Anke Marit Albers, Marcel Brass. 2012. The what and how components of cognitive control. NeuroImage 63, 203-211. [CrossRef]

7. André Vandierendonck, Jelle Demanet, Baptist Liefooghe, Frederick Verbruggen. 2012. A chain-retrieval model for voluntary task switching. Cognitive Psychology 65, 241-283. [CrossRef] 